\title{
Assessing the Intensity of Dog Walking and Impact on Overall Physical Activity: A Pilot Study Using Accelerometry
}

\author{
Elizabeth A. Richards ${ }^{1}$, Philip J. Troped ${ }^{2}$, Eunjung Lim ${ }^{3}$ \\ ${ }^{1}$ School of Nursing, Purdue University, West Lafayette, IN, USA \\ ${ }^{2}$ Department of Exercise and Health Sciences, University of Massachusetts, Boston, MA, USA \\ ${ }^{3}$ John A. Burns School of Medicine, University of Hawaii, Honolulu, HI, USA \\ Email: erichards@purdue.edu, phil.troped@umb.edu, lime@hawaii.edu
}

Received 14 May 2014; revised 13 June 2014; accepted 28 June 2014

Copyright (C) 2014 by authors and Scientific Research Publishing Inc.

This work is licensed under the Creative Commons Attribution International License (CC BY). http://creativecommons.org/licenses/by/4.0/

(c) (†) Open Access

\begin{abstract}
Introduction: Despite a growing body of research indicating that dog walking contributes to meeting physical activity (PA) guidelines, this literature is limited by the use of self-report measures of dog walking and overall PA. The objectives of this pilot study were to objectively assess dog walking with accelerometry, characterize the frequency, duration, and intensity of dog walking, and determine the contribution of dog walking to overall moderate-vigorous physical activity (MVPA). Methods: Sixty-five dog owners wore an Actigraph GT3X accelerometer for up to 7 consecutive days and recorded start/end times for dog walks with daily log sheets. Each minute with an activity count $\geq \mathbf{7 6 0}$ was classified as MVPA. Descriptive statistics were used to summarize all variables. Logistic regression was used to examine associations between dog walking MVPA bouts and meeting PA guidelines, controlling for age, education, income, and gender. Results: Participants walked their dog an average of $1.2 \pm 1.1$ times/day, averaged $28.0 \pm 15.6$ minutes/walk, and accumulated $22.9 \pm 17.5$ minutes of MVPA/day during dog walks, of which $21.7 \pm 17.9$ minutes were accumulated in bouts $\geq 10$ minutes. Seventy-eight percent of dog walking was classified as moderate-intensity and $3.5 \%$ was vigorous. Dog walking MVPA had a statistically significant positive association with meeting PA guidelines $(O R=2.32 ; 95 \% C I=1.06,5.08)$. Conclusions: The majority of dog walking minutes were moderate-intensity and most minutes of MVPA during dog walking occurred in bouts. These findings suggest that dog walking is consistent with current PA guidelines for adults and should receive more consideration as a PA promotion strategy.
\end{abstract}

\section{Keywords}

Physical Activity, Exercise, Pets, Physical Activity Assessment, Accelerometry

How to cite this paper: Richards, E.A., Troped, P.J. and Lim, E. (2014) Assessing the Intensity of Dog Walking and Impact on Overall Physical Activity: A Pilot Study Using Accelerometry. Open Journal of Preventive Medicine, 4, 523-528. 


\section{Introduction}

Participation in regular physical activity decreases the risk of many chronic diseases and conditions such as cardiovascular disease, type 2 diabetes mellitus, osteoporosis, obesity, breast and colon cancer, and falls in older adults [1]. Despite the well-known physical and mental health benefits of an active lifestyle, the proportion of US adults who meet physical activity guidelines remains low [1] [2]. Promotion of moderate-intensity physical activity, such as brisk walking, may be one the best types of physical activities to encourage, since it is inexpensive, does not require specific skills or abilities to perform, and has a low risk of injury [3]. Promotion of dog walking could be a promising strategy to increase walking and overall physical activity. Close to $50 \%$ of US households own a dog [4] and studies consistently show that this population has the potential to increase their physical activity via dog walking [5]-[7]. Approximately 40\% - 60\% of dog owners do not walk their dog at all or do not walk enough to meet US physical activity guidelines [6]-[8]. Furthermore, dog owners who do walk with their dog tend to be more active overall and are more likely to meet US physical activity guidelines than dog owners who do not walk with their dog [5].

Despite this evidence, little is known about how much of the physical activity obtained during dog walking is moderate-vigorous intensity physical activity (MVPA). One study examining dog behavior during walks suggests that a significant proportion of the dog's time is spent sniffing, which may result in stationary or slow walking speeds for owners [9]. A key limitation of dog walking studies is the use of self-report measures. Limitations of self-report measures include the potential for social desirability bias which can lead to over-reporting [10]. Furthermore, recalling physical activity is considered a complex cognitive task which may limit its effectiveness in youth and older adults [10]. The use of objective measures of physical activity, such as accelerometers addresses some of the limitations of self-report physical activity assessment [11]. For several years, researchers have been recommending that studies objectively measure the duration, frequency, and intensity of dog walking and the contribution this makes to overall physical activity [5]. To our knowledge, no published studies have objectively assessed these parameters with accelerometers. Therefore, the proportion of dog walking that is considered health-enhancing, specifically activity that is at least moderate-intensity and occurring in bouts $\geq 10$ minutes, is not known [1]. The objectives of this pilot study were to: 1) objectively assess the extent to which dog walking is comprised of moderate intensity bouts $\geq 10$ minutes; 2 ) assess differences in dog walking intensity, frequency, and duration by socio-demographic characteristics; and 3) objectively assess the contribution of dog walking to overall MVPA and meeting physical activity guidelines (150 minutes of MVPA/week accumulated in bouts $\geq 10$ minutes) [1].

\section{Methods}

\subsection{Participants}

This observational study was conducted in the fall of 2012 at Purdue University in Tippecanoe County, Indiana [12]. Dog owners eighteen years of age and older were recruited via a university-wide e-mail newsletter sent to all faculty, staff, and graduate students at the university. The primary inclusion criterion was that participants reported dog walking at least once per week. Ninety-three adults provided contact information. Sixty-seven individuals met with research staff and provided informed consent. One participant left the study for personal reasons and one was excluded due to non-compliance with the study protocol, resulting in a final sample of 65 participants. Study procedures were approved by the Purdue University Committee on the Use of Human Research Subjects.

\subsection{Equipment and Data Collection}

Participants wore an Actigraph GT3X [13] accelerometer, a lightweight monitor that captures acceleration in three planes. Accelerometers were initialized to collect data in 2-second epochs. Research staff met participants 1-3 days before monitoring to distribute accelerometers and describe study procedures. Participants received log sheets for the 7 monitoring days and were instructed to record the time they put the monitor on and took it off each day and the start and end times for each dog walk. Participants were instructed to wear the accelerometer on their right hip using an adjustable nylon belt, except when sleeping, bathing or swimming. After the monitoring period, research staff met participants to collect the devices and review the log sheets.

Participants also completed a brief survey. Demographic items included age, gender, race, ethnicity, highest level of education, marital status, and annual household income. Participants also reported height and weight. 
Body mass index (BMI) was calculated using the following formula: weight (lb)/[height (in)] $]^{2} \times 703$ [14]. Participants were classified as overweight if BMI was 25.0 - 29.9 and obese if BMI was $\geq 30.0$.

\subsection{Data Processing}

Accelerometer data were downloaded, re-integrated up to 1-minute, and screened for valid wear time using ActiLife $5^{\circledR}$ software [13]. Invalid wear time was defined as 60 minutes of consecutive zeros with a 2 minute spike tolerance [13]. Valid monitoring days were defined as having $\geq 600$ minutes of valid wear time and participants were required to have $\geq 4$ valid days to be included in the analysis [15]. Matthews' 2005 cut-points were used to classify intensity for each minute: $<100=$ sedentary; $100-759=$ light; $760-5724=$ moderate; and $\geq 5725=$ vigorous [16] [17]. These cut-points were chosen based on a recent validation study of free-living activity that compared the 2006 and 2010 Crouter algorithms, the National Health and Nutrition Examination Survey cut-points, and the Mathews cut-points for Actigraph data and which showed that among the four approaches the Matthews cut-points had the lowest mean bias in overestimating MVPA when compared to indirect calorimetry [16]. Using the times indicated for dog walking on the daily log sheets, each accelerometer minute was coded for dog walking. Bouts of dog walking and overall MVPA were calculated using National Health and Nutrition Examination Survey SAS code [18].

\subsection{Statistical Analysis}

Descriptive statistics were used to summarize all variables. One-way ANOVA was used to detect differences in dog walking outcomes (e.g., intensity, frequency, duration) by participant socio-demographic characteristics. Paired t-tests were used to compare the means of weekday versus weekend activity. Logistic regression was used to examine the association between dog walking MVPA bouts and meeting physical activity guidelines, controlling for age, education, income, and gender. Analyses were completed using SAS $^{\circledR} 9.3$ [19].Statistical significance was set at $p<0.05$.

\section{Results}

\subsection{Participant and Monitoring Characteristics}

Participants' mean age was $39.0 \pm 13.0$ years and $71 \%$ were female. Overall, participants were well educated and $45 \%$ were classified as overweight or obese. Fifty-nine percent $(n=38)$ had 7 days of valid monitoring; $26 \%$ $(n=17)$ had 6 days; $11 \%(n=7)$ had 5 days; and $5 \%(n=3)$ had 4 days. Valid wear time averaged $846.5 \pm 68.8$ minutes/day.

\subsection{Physical Activity and Dog Walking}

On average participants engaged in $106.8 \pm 35.9$ minutes/day of accumulated MVPA and $50.8 \pm 29.9$ minutes were obtained in bouts of 10 minutes or more (Table 1). Participants walked their dog an average of $1.2 \pm 1.1$ times per day and averaged $28.0 \pm 15.6$ minutes per walk. During dog walks, participants accumulated $22.9 \pm$ 17.5 minutes of MVPA/day, of which $21.7 \pm 17.9$ minutes were in bouts $\geq 10$ minutes. Mean daily minutes of dog walking were approximately 9 minutes longer on weekends versus weekdays, though there were no significant differences in the MVPA obtained during dog walks on weekends versus weekdays (Table 1).

As shown in Table 2, there were no significant differences in dog walking MVPA by gender, race, income, education, or weight status. Daily MVPA from dog walking among adults 55 years of age and older was two-fold greater than that of participants thirty years old or younger $(p=0.02)$.

Overall, 3.8\% of dog walking was classified as sedentary, $14.4 \%$ was light intensity, $78.3 \%$ was moderate intensity and 3.5\% was vigorous intensity. Of the MVPA obtained during dog walking, 95\% of the minutes occurred in bouts $\geq 10$ minutes. In addition, dog walking MVPA bouts accounted for $43 \%$ of all MVPA accumulated in bouts. MVPA during dog walking (in bouts) had a statistically significant positive association with meeting physical activity guidelines $(\mathrm{OR}=2.32 ; 95 \% \mathrm{CI}=1.06-5.08)$.

\section{Discussion}

This study found that a significant proportion of dog walking is MVPA occurring in bouts $\geq 10$ minutes, indi- 
Table 1. Overall physical activity and dog walking, $2012(n=65)$.

\begin{tabular}{ccccc}
\hline Variable & Overall & Weekday & Weekend & $p^{a}$ \\
\hline Motal MVPA minutes/day & Mean \pm SD & Mean \pm SD & Mean \pm SD \\
Accumulated & & & & \\
Bouts $\geq 10$ minutes & $106.8 \pm 35.9$ & $104.5 \pm 36.6$ & $109.8 \pm 48.5$ & 0.20 \\
Dog walks/day & $50.8 \pm 29.9$ & $47.5 \pm 30.3$ & $58.6 \pm 42.8$ & 0.01 \\
Minutes per dog walk & $1.2 \pm 1.1$ & $1.2 \pm 1.1$ & $1.2 \pm 1.2$ & 0.58 \\
Dog walking MVPA minutes/day & $28.0 \pm 15.6$ & $26.1 \pm 15.2$ & $34.8 \pm 24.2$ & 0.01 \\
Accumulated & & & $25.9 \pm 25.1$ & 0.11 \\
Bouts $\geq 10$ minutes & $22.9 \pm 17.5$ & $21.8 \pm 17.1$ & $24.4 \pm 25.6$ & 0.12 \\
\hline
\end{tabular}

MVPA = moderate-to-vigorous physical activity; $\mathrm{SD}=$ standard deviation; ${ }^{\mathrm{a}} \mathrm{T}$-test used to compare means on weekdays and weekends.

Table 2. Daily minutes of dog walking MVPA by participant characteristics, $2012(n=65)$.

\begin{tabular}{|c|c|c|c|c|}
\hline Variable & $N$ & $\%$ & Daily minutes of dog walking MVPA (mean \pm SD) & $p^{\mathrm{a}}$ \\
\hline Gender & & & & 0.49 \\
\hline Male & 19 & 29.2 & $24.0 \pm 17.5$ & \\
\hline Female & 46 & 70.8 & $20.7 \pm 18.2$ & \\
\hline Age (yr.) & & & & 0.02 \\
\hline$\leq 30$ & 24 & 36.9 & $15.5 \pm 17.9$ & \\
\hline $31-54$ & 31 & 47.7 & $22.3 \pm 14.9$ & \\
\hline$\geq 55$ & 10 & 15.4 & $34.4 \pm 21.0$ & \\
\hline Race & & & & 0.76 \\
\hline White/Caucasian & 57 & 87.7 & $22.0 \pm 17.1$ & \\
\hline Other & 8 & 12.3 & $19.8 \pm 26.0$ & \\
\hline Weight status & & & & 0.19 \\
\hline Normal & 36 & 55.4 & $23.9 \pm 17.7$ & \\
\hline Overweight & 17 & 26.2 & $23.0 \pm 21.0$ & \\
\hline Obese & 12 & 18.5 & $13.1 \pm 11.7$ & \\
\hline Income $^{\mathrm{b}}$ & & & & 0.21 \\
\hline$<\$ 50,000$ & 25 & 38.5 & $18.3 \pm 18.5$ & \\
\hline$\$ 50,000$ - $\$ 89,999$ & 16 & 24.6 & $18.6 \pm 20.6$ & \\
\hline$\geq \$ 90,000$ & 17 & 26.2 & $27.5 \pm 13.2$ & \\
\hline Education & & & & 0.48 \\
\hline High school or technical/trade school & 5 & 7.7 & $12.0 \pm 7.3$ & \\
\hline 2 - 4 year college & 27 & 41.5 & $24.7 \pm 21.5$ & \\
\hline Masters or professional degree & 20 & 30.8 & $19.6 \pm 16.0$ & \\
\hline Doctoral degree & 13 & 20.0 & $22.0 \pm 14.9$ & \\
\hline
\end{tabular}

MVPA = moderate-to-vigorous physical activity; $\mathrm{SD}=$ standard deviation; ${ }^{\mathrm{a} O n e-w a y ~ A n a l y s i s ~ o f ~ v a r i a n c e ~ p e r f o r m e d ~ t o ~ a s s e s s ~ d i f f e r e n c e s ~ w i t h ~ s t a t i s-~}$ tical significance set at $p<0.05$; ${ }^{b}$ Data does not equal $100 \%$ due to missing responses. 
cating this activity could be a legitimate form of health-enhancing physical activity, particularly among older adults. Furthermore, over $40 \%$ of MVPA accumulated during bouts occurred during dog walking, suggesting participants may use dog walking as a form of structured exercise. These results, albeit on a small sample, indicate that promotion of dog walking could be a viable approach for increasing physical activity in the US since it is estimated that $47 \%$ of households own a dog and up to $60 \%$ of dog owners do not walk their dog at levels sufficient to achieve health benefits [4] [6].

Promotion of dog walking could also be an effective strategy to combat obesity on a population level through additional energy expenditure. One metabolic equivalent (MET) is defined as 1 kilocalorie (kcal) per kilogram of body weight per hour and approximates the energy cost of quiet sitting. The MET value for dog walking is 3.0 [20]. Assuming energy intake remains constant, an average US man (at 195.5 pounds) and average woman (at 166.2 pounds) would expend $931.1 \mathrm{kcal} /$ week and $791.6 \mathrm{kcal} /$ week, respectively, if they walked their dog 30 minutes each day [21]. Over one year, this amount of dog walking could yield a weight loss of 14.2 pounds for men and 12.1 pounds for women [22]. Thus dog walking could significantly contribute to weight loss and weight maintenance.

Strengths of this study include the use of an objective measure of physical activity. To our knowledge, this is the first study to assess physical activity during dog walking with accelerometers. Limitations of this study include a small convenience sample that is predominantly comprised of white, well-educated females. Thus generalization to other US dog owners should be made cautiously. Furthermore, this study was conducted in the fall when weather was favorable for walking. However, two studies have shown that dog walkers are more likely to participate in outdoor recreational walking during inclement and winter weather than non-dog walkers [23] [24]. This suggests that seasonal effects may not be as pronounced with dog walking compared to other forms of outdoor recreational physical activity which tends to decrease during the winter months [25]. Also, the use of relatively lower activity cut-points for moderate physical activity may have led to overestimating moderate-intensity dog walking and the contribution this makes to meeting physical activity guidelines. However, as noted previously, recent research indicates that these cut-points introduce the least amount of bias in overestimating physical activity when compared to indirect calorimetry [16].

Further dog walking studies using accelerometry are needed to confirm whether dog walking is primarily a moderate intensity activity and substantially contributes to overall MVPA. In addition, future longitudinal research is needed to explore whether dog walking typically increases individuals' total physical activity or substitutes for other forms of physical activity, thereby not increasing total physical activity.

Though further research is needed to replicate the results in larger and more diverse populations, the findings from this small pilot study suggest that dog walking is a type of physical activity that merits greater attention from public health officials and practitioners. Increasing the prevalence of dog walking could help the US attain physical activity objectives within Healthy People 2020, specifically addressing the prevalence of leisure-time physical activity.

\section{References}

[1] Physical Activity Guidelines Advisory Committee Report (2008) US Department of Health and Human Services. http://www.health.gov/paguidelines/Report/pdf/CommitteeReport.pdf

[2] Centers for Disease Control and Prevention (2013) Early Release of Selected Estimates Based on Data from the 2012 National Health Interview Survey: Leisure-Time Physical Activity.

http://www.cdc.gov/nchs/nhis/released201306.htm\#7

[3] Hootman, J. Macera, C., Ainsworth, B., Martin, M., Addy, C. and Blair, S. (2011) Association among Physical Activity Level, Cardiorespiratory Fitness, and Risk of Musculoskeletal Injury. American Journal of Epidemiology, 54, $251-258$.

[4] The Humane Society of the United States (2013) US Pet Ownership Statistics. http://www.humanesociety.org/issues/pet_overpopulation/facts/pet_ownership_statistics.html\#.Uqc4hE2A05s

[5] Christian, H.E., Westgarth, C., Bauman, A., Richards, E.A., Rhodes, R.E., Evenson, K.R., et al. (2013) Dog Ownership and Physical Activity: A Review of the Evidence. Journal of Physical Activity and Health, 10, 750-759.

[6] Reeves, M.J., Rafferty, A.P., Miller, C.E. and Lyon-Callo, S.K. (2011) The Impact of Dog Walking on Leisure-Time Physical Activity: Results from a Population-Based Survey of Michigan Adults. Journal of Physical Activity and Health, 8, 436-444.

[7] Richards, E.A., McDonough, M., Edwards, N., Lyle, R. and Troped, P.J. (2013) Psychosocial and Environmental Factors Associated with Dog Walking. International Journal of Health Promotion and Education, 51, 198-211. 
http://dx.doi.org/10.1080/14635240.2013.802546

[8] Bauman, A.E., Russell, S.J., Furber, S.E. and Dobson, A.J. (2001) The Epidemiology of Dog Walking: An Unmet Need for Human and Canine Health. Medical Journal of Australia, 175, 632-634.

[9] Westgarth, C., Christley, R.M., Pinchbeck, G.L., Gaskell, R.M., Dawson, S. and Bradshaw, J.W.S. (2011) Dog Behaviour on Walks and the Effect of Use of the Leash. Applied Animal Behaviour Science, 125, 38-46. http://dx.doi.org/10.1016/j.applanim.2010.03.007

[10] Sallis, J. and Saelens, B. (2000) Assessment of Physical Activity by Self-report: Status, Limitations, and Future Directions. Research Quarterly for Exercise Sport, 71, S1-S14.

[11] Troiano, R.P. (2005) Timely Meeting: Objective Measurement of Physical Activity. Medicine Science in Sports Exercise, 37, S487-489. http://dx.doi.org/10.1249/01.mss.0000185473.32846.c3

[12] United States Census Bureau (2014) Tippecanoe County, Indiana: State and County Quick Facts. http://quickfacts.census.gov/qfd/states/18/18157.html

[13] (2010) ActiLife 5 User's Manual. Actigraph Corporation, Pensacola.

[14] Centers for Disease Control and Prevention (2011) About BMI for Adults. http://www.cdc.gov/healthyweight/assessing/bmi/adult bmi/index.html\#Interpreted

[15] Troiano, R.P., Berrigan, D., Dodd, K.W., Masse, L.C., Tilert, T. and McDowell, M. (2008) Physical Activity in the United States Measured by Accelerometer. Medicine Science in Sports Exercise, 40, 181-188. http://dx.doi.org/10.1249/mss.0b013e31815a51b3

[16] Crouter,S.E., DellaValle, D.M., Haas, J.D., Frongillo, E.A. and Bassett, D.R. (2013)Validity of Actigraph 2-Regression Model, Matthews Cut-Points, and NHANES Cut-Points for Assessing Free-Living Physical Activity. Journal of Physical Activity and Health, 10, 504-514.

[17] Matthews, C.E. (2005) Calibration of Accelerometer Output for Adults. Medicine Science in Sports, 37, S512-522.

[18] National Institutes of Health (2013) SAS Programs for Analyzing NHANES 2003-2004 Accelerometer Data. http://appliedresearch.cancer.gov/tools/nhanes_pam/

[19] (2009) SAS/STAT 9.2 User’s Guide. 2nd Edition, SAS Institute, Cary.

[20] Ainsworth, B.E., Haskell, W.L., Whitt, M.C., Irwin, M.L., Swartz, A.M., Strath, S.J., et al. (2000) Compendium of Physical Activities: An Update of Activity Codes and MET Intensities. Medicine Science in Sports, 32, S498-S504.

[21] Fryar, C.D., Gu,Q. and Ogden, C.L. (2012) Anthropometric Reference Data for Children and Adults: United States, 2007-2010. Vital and Health Statistics Series, 11, 1-40.

[22] Hall, K.D. (2007)What is the Required Energy Deficit per Unit Weight Loss? International Journal of Obesity, 32,573-576. http://dx.doi.org/10.1038/sj.ijo.0803720

[23] Lail, P., McCormack, G. and Rock, M. (2011) Does Dog-Ownership Influence Seasonal Patterns of Neighbourood-Based Walking among Adults? A Longitudinal Study. BMC Public Health, 11, 148. http://dx.doi.org/10.1186/1471-2458-11-148

[24] Temple, V., Rhodes, R. and Wharf Higgins, J. (2011) Unleashing Physical Activity: An Observational Study of Park Use, Dog Walking, and Physical Activity. Journal of Physical Activity and Health, 8, 766-774.

[25] Tucker, P. and Gilliland J. (2007) The Effect of Season and Weather on Physical Activity: A Systematic Review. Public Health, 121, 909-922. http://dx.doi.org/10.1016/j.puhe.2007.04.009

\section{Abbreviations}

BMI: body mass index

Kcal: kilocalorie

MET: metabolic equivalent

MVPA: moderate to vigorous intensity physical activity

PA: physical activity 